\title{
Simple model of bulk and surface excitation effects to inelastic scattering in low-energy electron beam irradiation of multi-walled carbon nanotubes
}

\author{
Ioanna Kyriakou, ${ }^{1}$ Dimitris Emfietzoglou, ${ }^{1, a)}$ Rafael Garcia-Molina, ${ }^{2}$ Isabel Abril, ${ }^{3}$ \\ and Kostas Kostarelos ${ }^{4}$ \\ ${ }^{1}$ Medical Physics Lab, University of Ioannina Medical School, 45110 Ioannina, Greece \\ ${ }^{2}$ Departamento de Física-CIOyN, Universidad de Murcia, E-30100 Murcia, Spain \\ ${ }^{3}$ Departament de Física Aplicada, Universitat d'Alacant, Apartat 99, E-03080 Alacant, Spain \\ ${ }^{4}$ Nanomedicine Lab, Centre for Drug Delivery Research, The School of Pharmacy, University of London, \\ London WCIN IAX, United Kingdom
}

(Received 8 July 2011; accepted 15 July 2011; published online 1 September 2011)

\begin{abstract}
The effect of bulk and surface excitations to inelastic scattering in low-energy electron beam irradiation of multi-walled carbon nanotubes (MWNTs) is studied using the dielectric formalism. Calculations are based on a semiempirical dielectric response function for MWCNTs determined by means of a many-pole plasmon model with parameters adjusted to available experimental spectroscopic data under theoretical sum-rule constrains. Finite-size effects are considered in the context of electron gas theory via a boundary correction term in the plasmon dispersion relations, thus, allowing a more realistic extrapolation of the electronic excitation spectrum over the whole energy-momentum plane. Energy-loss differential and total inelastic scattering cross sections as a function of electron energy and distance from the surface, valid over the energy range $\sim 50-30,000$ $\mathrm{eV}$, are calculated with the individual contribution of bulk and surface excitations separated and analyzed for the case of normally incident and escaping electrons. The sensitivity of the results to the various approximations for the spatial dispersion of the electronic excitations is quantified. Surface excitations are shown to have a strong influence upon the shape and intensity of the energy-loss differential cross section in the near surface region whereas the general notion of a spatially invariant inelastic mean free path inside the material is found to be of good approximation. (C) 2011 American Institute of Physics. [doi:10.1063/1.3626460]
\end{abstract}

\section{INTRODUCTION}

Since the pioneering work of Iijima, ${ }^{1}$ carbon nanotubes (CNTs) have become one of the most promising materials in nanotechnology research. ${ }^{2}$ The study of charged particle interactions with CNTs is important for at least two reasons. First, for developing effective methodologies for the controlled-modification of their structure via electron- or ionbeam irradiation, ${ }^{3,4}$ and secondly, for predicting radiation damage in CNT-based devices to be used in space, ${ }^{5,6}$ nuclear technology applications (including nuclear medicine applications). ${ }^{7,8}$ For high-energy electron beams such as those employed in transmission electron microscopy (TEM), irradiation effects are mainly due to knock-on displacement of carbon atoms following elastic electron-nucleus scattering. ${ }^{9}$ These effects are relatively well-understood and are known to set in for electron beam energies above the socalled "knock-on threshold" which for CNTs is $\sim 80 \mathrm{keV}$ (the exact value depends on the CNT diameter). ${ }^{10-12}$ On the other hand, for low-energy electron beams (i.e., with energies below the knock-on threshold) irradiation effects in CNTs are due to electronic excitations induced by inelastic electron-electron scattering. It is now clear that low-energy electron-beam irradiation in scanning electron microscopy (SEM) or electron beam lithography (EBL), can efficiently alter CNT properties as a result of local chemical reactions

${ }^{\text {a)} E l e c t r o n i c ~ m a i l: ~ d e m f i e t z @ c c . u o i . g r . ~}$ mediated by the presence of gas radicals. ${ }^{13-17}$ Direct inelastic effects in CNTs have also been reported (see Ref. 18, and references therein) but the mechanism is still not clear ${ }^{19-21}$ given that CNTs are either metallic or narrow-gap semiconductors with delocalized electronic excitations having relatively short relaxation times. On the other hand, in lowdimensional systems the exact mechanism of the conversion of electronic excitation energy to nucleus kinetic energy may differ from that of bulk solids leading possibly to longer relaxation time of electronic excitations. ${ }^{22}$

Inelastic scattering of low-energy electrons is also employed in the characterization and fabrication of CNTbased Field-Effect-Transistors (CNT-FETs) (Ref. 23-25) and stimulated field-emission devices ${ }^{26-29}$ using SEM or EBL, as well as in the quantitative interpretation of spectroscopic data from surface-sensitive techniques, such as X-ray photoelectron spectroscopy (XPS), Auger-electron spectroscopy (AES) and reflection-electron-energy-loss spectroscopy (REELS). ${ }^{30,31}$ Finally, inelastic scattering of low-energy electrons is also important in understanding irradiation effects by high-energy ion beams ${ }^{32-34}$ or from various X-ray sources $^{35,36}$ due to the role of secondary electron cascades in the spatial distribution of energy absorption in the material.

First-principles calculations of the inelastic interaction of charged particles with CNTs have been undertaken ${ }^{37-42}$ that highlight finite-size and collective-excitation effects arising from the reduced dimensionality of the system. On the other hand, more simple models for the inelastic 
interaction of low-energy electrons $(<30 \mathrm{keV})$ with multiwalled carbon nanotubes (MWCNTs) have been employed for understanding the cutting mechanism of MWCNTs in a SEM $^{13-16}$ and also to carry out Monte Carlo electron trajectory simulations in MWCNT systems. ${ }^{28,43}$ One of the challenges in such efforts was to account within a simple model for the inelastic scattering properties of MWCNTs, rather than relying on general characteristics of electron beam-bulk matter interactions and/or graphite excitation properties.

Inspired by the successful use of optical data - first suggested by Powell ${ }^{44}$ - to inelastic scattering calculations for bulk solids and solid surfaces (as reviewed in Ref. 45) and, more recently, for nanostructures, ${ }^{46}$ here we describe a semiempirical model for the dynamic and non-local dielectric response function of MWCNTs that is used to calculate the contribution of bulk and surface excitations in the inelastic interaction of low-energy electrons $(<30 \mathrm{keV})$ with MWCNTs. Recently, we have presented calculations for electron- and proton-beam irradiation of CNTs based on an optical-data model and the Bethe approximation. ${ }^{47,48}$ This work is extended here in several aspects: (i) in view of new experimental data ${ }^{49}$ specific to MWCNTs for the momentum-dependence of the dielectric function, we examine different dispersion relations for the plasmon energy and linewidth; (ii) we consider energy losses to both bulk and surface excitations in a self-consistent manner within a single model; and (iii) the variation of inelastic scattering cross section with distance from the surface and electron direction is examined. Atomics units $\left(m_{e}=e=\hbar=1\right)$ are used throughout except where otherwise stated.

\section{DIELECTRIC RESPONSE FUNCTION}

In the present context, the spectrum of electronic excitations in MWCNTs is codified by the so-called energy-lossfunction (ELF) - not to be confused with the electronenergy-loss-spectrum (EELS) measured in a transmission experiment; although related the two magnitudes are not identical. ${ }^{50}$ Within the continuum-medium approximation which has been shown to work well even for nanostructures ${ }^{51}$ the ELF is defined as $\operatorname{Im}\{-1 / \varepsilon(\omega, k)\}$ where $\varepsilon(\omega, k)$ is the dielectric response function of the system with $\hbar \omega$ and $\hbar k$ being the energy- and momentum-transfer, respectively. It is well known that the dielectric response of CNT is anisotropic $^{52-55}$ and, in principle, a tensor description of its dielectric function is required, similar to the in-plane $\left(\varepsilon_{\perp}\right)$ and outof-plane $\left(\varepsilon_{\|}\right)$dielectric tensors of graphite. ${ }^{56}$ However, due to the cylindrical geometry of nanotubes, for randomly-oriented electron trajectories both the parallel and perpendicular components will co-exist and the same will also hold for bulk samples containing non-aligned CNTs (Ref. 57). Thus, in the present context, it is computationally convenient, as a first approximation, to loosely associate $\varepsilon(\omega, k)$ with an "effective" dielectric function averaged over all orientations.

Following the general procedure of the optical-data method, the analytic description of the ELF of MWCNTs proceeds in two steps in which one first determines the $\omega$-dependence of ELF from optical data (i.e., at vanishing momentum transfer, $k \approx 0$ ) and subsequently implements a suitable extension algorithm to describe the ELF for arbitrary values of momentum transfer $(k \neq 0)$.

\section{A. Optical limit $(k \rightarrow 0)$}

At vanishing $k$, the ELF of the outer-shell electrons (i.e., valence electrons) is based on the EELS measurements of Kuzuo and co-workers ${ }^{58}$ on a relatively thick MWCNT (44 walls and $31 \mathrm{~nm}$ in diameter) that cover the energy-loss range from threshold up to $50 \mathrm{eV}$. For energy losses higher than $\sim 100 \mathrm{eV}$ we can safely neglect solid-state effects and use the approximation ${ }^{50} \operatorname{Im}\{-1 / \varepsilon(\omega, k=0)\} \cong \operatorname{Im}\{\varepsilon(\omega$, $k=0)\}=c \mu / \omega$ where $c$ is the speed of light and $\mu$ is the $\mathrm{X}$ ray attenuation coefficient of carbon atoms. The latter is here obtained from the FFAST database of NIST (Ref. 59). Following Ritchie and Howie ${ }^{60}$ the EELS and X-ray data can be conveniently parameterized using a superposition of Drudetype ELFs as follows:

$$
\begin{aligned}
& \operatorname{Im}\left\{-\frac{1}{\varepsilon(\omega, k \approx 0)}\right\}_{\mathrm{data}}=\operatorname{Im}\left\{-\frac{1}{\varepsilon(\omega, k \approx 0)}\right\}_{\mathrm{EELS}} \\
& +\operatorname{Im}\{\varepsilon(\omega, k \approx 0)\}_{\mathrm{X}-\mathrm{ray}} \approx \sum_{i=1}^{m} \frac{A_{i}}{\omega_{i, 0}^{2}} \\
& \quad \times \operatorname{Im}\left\{-\frac{1}{\varepsilon_{\mathrm{D}}\left(\omega, k=0 ; \omega_{i, 0}, \gamma_{i, 0}\right)}\right\} \Theta\left(\omega-\omega_{\mathrm{th}, i}\right)
\end{aligned}
$$

whereas $\Theta(\ldots)$ represents the Heaviside step function. The ELF of the Drude-type dielectric function, $\varepsilon_{\mathrm{D}}$, has the form:

$$
\operatorname{Im}\left\{-\frac{1}{\varepsilon_{\mathrm{D}}\left(\omega, k=0 ; \omega_{i, 0}, \gamma_{i, 0}\right)}\right\}=\frac{\omega_{i, 0}^{2} \gamma_{i, 0} \omega}{\left[\left(\omega_{i, 0}^{2}-\omega^{2}\right)^{2}+\left(\gamma_{i, 0} \omega\right)^{2}\right]}
$$

The above description essentially generalizes the plasmonpole approximation of the electron gas to a many-pole plasmon model by considering an $m$ number of poles that correspond to sub-bands each of which is associated with a "bound" plasmon (i.e., a collective-like excitation) of strength $A_{i}$, frequency $\omega_{i, 0}$, linewidth (or damping) $\gamma_{i, 0}$, and threshold $\omega_{\mathrm{th}, i}$; the subscript " 0 " stands for $k=0$. Note that whereas one can practically assume here $\omega_{\mathrm{th}, i} \approx 0$ for the valence excitations, the condition $\omega_{\text {th }, i} \neq 0$ should be used for the inner-shell (here the carbon K-shell) electrons. The $4 m$ parameters $\left\{A_{i}, \omega_{i, 0}, \gamma_{i, 0}, \omega_{\mathrm{th}, i}\right\}$ of the many-pole plasmon model are determined empirically via Eq. (1) (Ref. 61). A comparison between our model ELF and the experimental data can be seen in Fig. 1. Our parametric ELF model with $m=13$ seems to provide a good overall representation of the experimental data over most of the important energy-loss range of interest here. Material-specific solid-state effects are evident below $50 \mathrm{eV}$ where the MWCNT data with the $\pi$ and $\pi+\sigma$ plasmon peaks at $\sim 6$ and $\sim 23 \mathrm{eV}$, respectively, begin to differ considerably from those of carbon. Well beyond the K-shell edge at $\sim 285 \mathrm{eV}$, Eq. (1) starts overestimating the data due to its wrong asymptotic behavior. ${ }^{48}$ However, this deficiency is inconsequential for inelastic cross section calculations given that the ELF has already decreased by almost six orders of magnitude. More accurate 


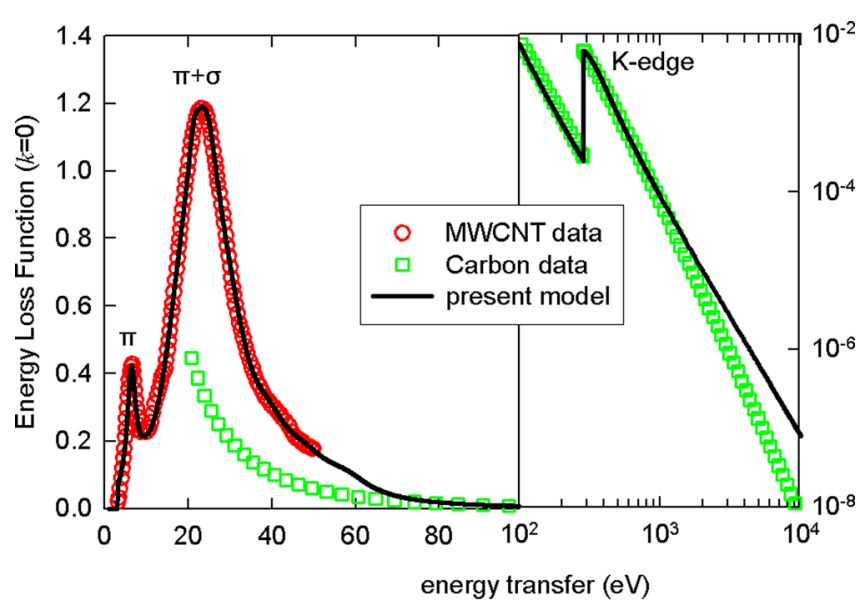

FIG. 1. (Color online) Comparison of our model energy loss function (ELF) at vanishing momentum transfer (i.e., optical limit, $k \approx 0$ ), described by Eqs. (1) and (2), with the experimental ELF deduced from the EELS data for MWCNTs of Ref. 58 and the X-ray atomic data for carbon of Ref. 59.

K-shell excitation functions might be needed though for stopping power calculations. ${ }^{48}$ The internal consistency of our model was tested using the (optical) $f$-sum rule and a form of the perfect screening sum rule called the KramersKronig or KK-sum rule: ${ }^{62}$

$f$-sum rule: $N_{\text {eff }}\left(\omega_{\max }\right)=\frac{1}{2 \pi^{2} n} \int_{0}^{\omega_{\max }} \omega \operatorname{Im}\left\{-\frac{1}{\varepsilon(\omega, k=0)}\right\} d \omega$

KK-sum rule: $P_{\text {eff }}\left(\omega_{\max }\right)=\frac{2}{\pi} \int_{0}^{\omega_{\max }} \frac{1}{\omega} \operatorname{Im}\left\{-\frac{1}{\varepsilon(\omega, k=0)}\right\} d \omega$ $+\operatorname{Re}\left\{\frac{1}{\varepsilon(\omega=0, k=0)}\right\}$

where $n=Z N_{\mathrm{Av}} \rho / M$ is the electronic density of the target, with $Z, \rho$, and $M$ being, respectively, the atomic number, mass density, and atomic weight $\left(N_{\mathrm{Av}}\right.$ is Avogadro's number). With respect to the calculation of the KK-sum rule from Eq. (3b), the value $\operatorname{Re}\{1 / \varepsilon(0,0)\}=1 / \operatorname{Re}\left\{\varepsilon_{1}(0,0)\right\}$ $\approx 0.205$ was deduced from the EELS data for the MWCNTs (Ref. 58). From inspection of Eqs. [3(a) and 3(b)] we can see that in $N_{\text {eff }}$ the ELF is weighted by $\omega$ whereas in $P_{\text {eff }}$ by $\omega^{-1}$. It follows that the main contribution to $N_{\text {eff }}$ would come from large energy losses whereas to $P_{\text {eff }}$ would come from small energy losses. Therefore, to ensure a good-quality ELF, it is important that both sum rules are fulfilled. A plot of $N_{\text {eff }}$ and $P_{\text {eff }}$ as a function of the upper limit of integration $\left(\omega_{\max }\right)$ is depicted in Fig. 2. It can be seen that both sum rules are satisfied almost exactly $( \pm 1 \%)$ by our model. In principle the $f$-sum rule of Eq. (3a) should be fulfilled for all $k$. The form of Eq. (2) ensures that if the $f$-sum rule is fulfilled for $k=0$ it would then be fulfilled for all $k$ as long as $\omega_{\text {th }, i} \rightarrow 0$. In our case, $\omega_{\text {th }, i} \approx 0$ for the valence shells but clearly not for the K-shell. Since the contribution of the Kshell to the inelastic scattering cross section in the present energy range is very small (less than a few percent) the above inconsistency is inconsequential for the present work. As also noted earlier, an improved model for the K-shell

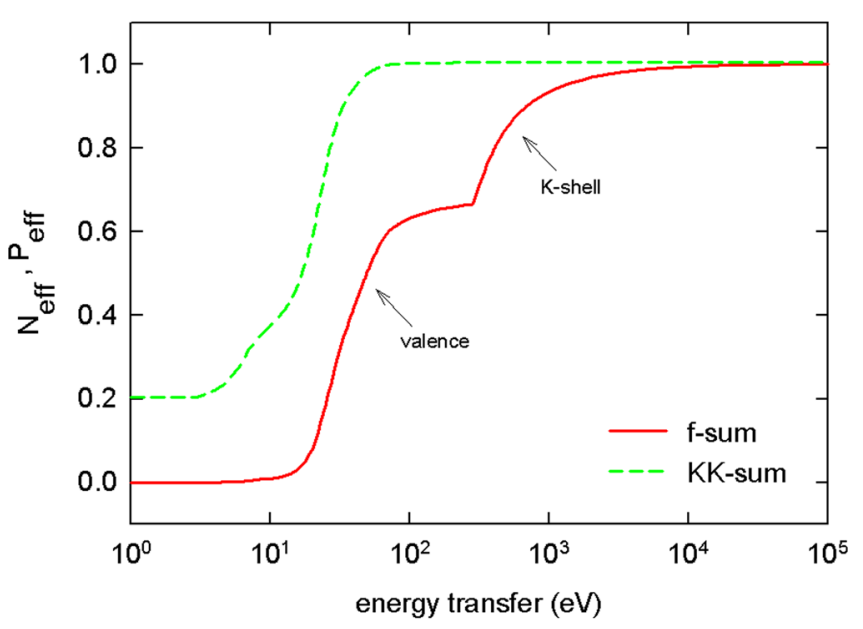

FIG. 2. (Color online) The $f$ - and KK-sum rules of our model ELF for MWCNTs computed from Eqs. (3a) and (3b), respectively, as a function of the upper limit of integration in energy transfer. The contribution of the valence and $\mathrm{K}$-shell excitations to the $f$-sum is indicated.

would be needed when extending to much higher electron energies (say to $100 \mathrm{keV}$ and above) or for carrying out stopping power calculations.

Given that MWCNTs can be produced in different sizes (e.g., different number of walls) and chiralities, it is natural to question the validity of our parameterized ELF (derived from a given set of experimental data ${ }^{58}$ ) for other MWCNT systems. The finite length of the MWCNT is of no concern here as long as it is very much larger than the diameter, which is most often the case. In contrast, the finite lateral size of the nanotube produces most of the interesting effects in the present context. The EELS measurements of Kuzuo and co-workers ${ }^{58}$ pertain to MWCNTs with diameter ranging from 13 to $34 \mathrm{~nm}$ (number of walls $21-44$ ) under penetrating beam geometry. The $\pi+\sigma$ plasmon peak of the different MWCNTs were found to range from 22.0 to $24.5 \mathrm{eV}$, in good agreement with the mean value for the in-plane and out-of-plane plasmon value of graphite. ${ }^{56,63,64}$ Subsequent studies ${ }^{52,65,66}$ have confirmed that the EELS data depicted in Fig. 1 are indeed representative of relatively thick MWCNTs with diameter larger than $\sim 10 \mathrm{~nm}$ (or more than $\sim 15$ walls). It is therefore justified to associate our model ELF, described at $k \approx 0$ by Eqs. (1) and (2), with the bulk ELF of mediumlarge diameter MWCNTs and, more generally, of macroscopic MWCNT samples.

On the other hand, the inelastic scattering of charged particle beams by surfaces gives rise to additional excitation modes (most notably surface plasmons) as first predicted by Ritchie $^{67}$ and experimentally confirmed by Powell and Swan. ${ }^{68}$ Moreover, the probability of surface plasmon excitations is known to increase with decreasing target thickness and beam energy; ${ }^{67}$ thus, making them particularly relevant to low-energy electron beam irradiation of nanostructures. In fact, TEM/EELS measurements in different CNT systems have revealed that the excitation spectrum at the near surface region exhibits a clear shift toward lower energy losses as compared to the bulk excitation spectrum depicted in Fig. 1 (Refs. 69-72). Specifically, the $\pi+\sigma$ plasmon peak is shifted to $\sim 15-17 \mathrm{eV}$, in very good agreement with Ritchie's 
surface plasmon frequency (Ref. 67) $\omega_{s p}=\omega_{v p} / \sqrt{2}$ where $\omega_{v p}=(4 \pi n)^{1 / 2}$ is the frequency of the volume (or bulk) plasmon. Although it is still not clear whether the observed shift of the plasmon energy of CNTs to lower energy losses should be attributed to a change in the strength of screening ${ }^{49}$ or to a surface plasmon, ${ }^{70}$ for modeling purposes it is convenient here to adopt the latter hypothesis. We therefore model the surface ELF of MWCNTs at the optical limit $(k \approx 0)$ by means of the bulk dielectric function using the relation: ${ }^{73}$

$$
\operatorname{Im}\left\{-\frac{1}{\varepsilon(\omega, k=0)}\right\}_{\text {surface }}=\operatorname{Im}\left\{-\frac{1}{\varepsilon(\omega, k=0)_{\text {bulk }}+1}\right\}
$$

The bulk dielectric function in the right-hand-side of Eq. (4) can be obtained from the following set of equations: ${ }^{.73}$

$$
\begin{gathered}
\varepsilon(\omega, k=0)=\varepsilon_{1}(\omega, k=0)+i \varepsilon_{2}(\omega, k=0) \\
\varepsilon_{1}(\omega, k=0)=\frac{\operatorname{Re}\{1 / \varepsilon(\omega, k=0)\}}{\operatorname{Re}\{1 / \varepsilon(\omega, k=0)\}^{2}+\operatorname{Im}\{1 / \varepsilon(\omega, k=0)\}^{2}} \\
\varepsilon_{2}(\omega, k=0)=\frac{-\operatorname{Im}\{1 / \varepsilon(\omega, k=0)\}}{\operatorname{Re}\{1 / \varepsilon(\omega, k=0)\}^{2}+\operatorname{Im}\{1 / \varepsilon(\omega, k=0)\}^{2}}
\end{gathered}
$$

The real part of $1 / \varepsilon(\omega, k=0)$ needed in Eq. (5b) and (5c) can be obtained from its imaginary part through the Kramers-Kronig relation: ${ }^{73}$

$$
\begin{aligned}
& \operatorname{Re}\left\{\frac{1}{\varepsilon(\omega, k=0)}\right\} \\
& \quad=1+\frac{2}{\pi} P \int_{0}^{\infty} \operatorname{Im}\left\{\frac{1}{\varepsilon\left(\omega^{\prime}, k=0\right)}\right\} \frac{\omega^{\prime}}{\omega^{/ 2}-\omega^{2}} d \omega^{\prime}
\end{aligned}
$$

where $P$ denotes the Cauchy principal part of the integral. The form of Eq. (2) used to describe $\operatorname{Im}\{-1 / \varepsilon(\omega, k=0)\}$ has the important advantage that, via Eq. (6), the $\operatorname{Re}\{1 / \varepsilon(\omega, k=0)\}$ can also be expressed analytically as follows:

$$
\begin{aligned}
& \operatorname{Re}\left\{\frac{1}{\varepsilon(\omega, k=0)}\right\} \\
& \quad=1-\sum_{i} \frac{A_{i}\left(\omega_{i, 0}^{2}-\omega^{2}\right)}{\left[\left(\omega_{i, 0}^{2}-\omega^{2}\right)^{2}+\left(\gamma_{i, 0} \omega\right)^{2}\right]} \Theta\left(\omega-\omega_{\mathrm{th}, i}\right)
\end{aligned}
$$

Inserting now Eqs. (2) and (7) into Eq. (5) we can calculate the bulk dielectric function which, in turn, determines the surface ELF by means of Eq. (4).

\section{B. Finite momentum transfer $(\boldsymbol{k} \neq 0)$}

Most theoretical studies on the inelastic scattering of charged particles in nanostructures (including thin films) use a non-dispersive (i.e., local) dielectric response function to simplify the numerical work. However, it is well-known that for sub-keV electrons (especially below $200 \mathrm{eV}$ ) inelastic scattering is sensitive to the $k$-dependence of the ELF
(Ref. 45). Moreover, in systems with strong spatial confinement (such as nanotubes) the $k$-dependence of the dielectric function, which reflects the spatial dispersion of the electronic excitations, is expected to play an important role. ${ }^{74}$ For MWCNTs in particular, the spatial confinement of the electronic subsystem has been shown to lead to a dimensionality crossover with increasing $k$, due to the coupling-decoupling of plasmon excitations in individual wall layers. ${ }^{75}$ Although rigorous solutions of the plasmon dispersion relation in cylindrical nanostructures (wires or tubes) have been presented recently, ${ }^{76-80}$ we here undertake the more modest task to heuristically develop — in the spirit of the opticaldata method — a computationally simple, yet physically motivated, analytic expressions that can be conveniently implemented into Eq. (1) following the Ritchie-Howie prescription. ${ }^{60}$ We should note that implicit in our calculations (and the discussion that follows) is the approximation that all excitation modes (denoted by the subscript $i$ ) considered in Eq. (1) share the same $k$-dependence which will be deduced here through considerations on the $\pi+\sigma$ plasmon dispersion. Although both the interband and $\pi$ plasmon excitations of CNTs are known to disperse differently from the $\pi+\sigma$ plasmon, ${ }^{81}$ the present simplification should suffice for inelastic scattering calculations with electron beam energies well exceeding the valence excitations of the system (i.e., above $\sim 50-100 \mathrm{eV}$ ) since the $\pi+\sigma$ plasmon peak dominates the ELF representing the main energy-loss channel of the system; as a matter of fact, this constitutes a standard approximation in optical-data model calculations above $\sim 50 \mathrm{eV}$ (Refs. 82 and 83).

\section{Influence of plasmon dispersion}

Perhaps the simplest approximation to the plasmon dispersion relation is to assume a quasi-two-body collision between the beam and target electrons leading to the wellknown expression: ${ }^{84}$

$$
\omega_{v p}(k)=\omega_{v p}+\frac{k^{2}}{2}
$$

Equation (8) is correct at the high- $k$ limit, where the ELF shows a maximum along the dispersion line $\omega \approx k^{2} / 2$, which is the so-called Bethe ridge. Thus, Eq. (8) is expected to work well for single-particle excitations which are dominant at high- $k$. On the other hand, for small- $k$ the wavelength of the perturbation commonly extends over a large number of atoms and collective excitations are more important than singleparticle excitations. Naturally then, the $k$-dependence (or spatial dispersion) of collective excitations would be strongly influenced by the dimensionality of the system. The Lindhard dielectric function of the three-dimensional electron gas (3DEG) predicts a (volume) plasmon dispersion relation that in the long wavelength limit (or small-k) reads: ${ }^{85}$

$$
\omega_{v p}^{(3 \mathrm{DEG})}(k)=\omega_{v p}+a k^{2}
$$

where the dispersion coefficient is $a=\beta^{2} / 2 \omega_{v p}$ with $\beta=\sqrt{3 / 5} v_{\mathrm{F}}$ and $v_{\mathrm{F}}$ being the Fermi velocity of the 3DEG 
which is solely a function of its electronic density. Then, by combining Eqs. (8) and (9) we obtain the familiar expres$\operatorname{sion}^{86}$ for infinite (i.e., unbounded) 3D media which in the present context reads:

$$
\omega_{i}^{(3 \mathrm{D})}(k)=\sqrt{\omega_{i, 0}^{2}+\beta^{2} k^{2}+k^{4} / 4}
$$

Equation (10) accounts, in an approximate manner, for both collective and single-particle excitations effective, respectively, at the low- and high- $k$ limits. Specifically, collective excitations contribute the quadratic term whereas single-particle excitations the quartic term (note that $k^{4} / 4$ equals the square of the kinetic energy of a free electron with wave vector $k$ ). An obvious shortcoming of Eq. (10) is that it does not account for boundary effects arising from the finite lateral size of the target. As discussed in the previous section, it is convenient for modeling purposes to attribute these effects to the excitation of surface plasmons. Assuming that curvature effects are small (which is a reasonable approximation for MWCNTs larger than $\sim 10 \mathrm{~nm}$ in diameter ${ }^{49,57}$ we will heuristically consider boundary corrections to Eq. (9) via two approximations, namely, the "bulk" planar-surface (BPS) model and the quasi-two-dimensional (Q2D) model. In the BPS model, boundary effects are accounted for through the surface plasmon dispersion of a semi-infinite electron gas (SIEG) that in the long wavelength limit reads: ${ }^{87}$

$$
\omega_{s p}^{(\mathrm{SIEG})}(k)=\omega_{s p}+a_{\mathrm{s}} Q
$$

where $Q$ is the component of $k$ parallel to the surface, $\omega_{s p}=\omega_{v p} / \sqrt{2}$ is the Ritchie frequency, and $a_{s}=\sqrt{3 / 20} v_{\mathrm{F}}$. Assuming now that the dielectric function is only weakly dependent upon the momentum transfer vector normal to the surface, i.e., $\varepsilon(\omega, k) \approx \varepsilon(\omega, Q)$, we can combine Eqs. (10) and (11) to arrive at the dispersion expression of the BPS model:

$$
\omega_{i}^{(\mathrm{BPS})}(k)=\sqrt{\omega_{i, 0}^{2}+\alpha k+\beta^{2} k^{2}+k^{4} / 4}
$$

where $\alpha=\sqrt{3 / 10} \omega_{v p} v_{\mathrm{F}}$. Thus, inclusion of boundary effects in the context of the BPS model results in the addition of a linear dispersion term $(\sim k)$ to the standard 3D expression.

On the other hand, in the Q2D model, surface excitations are obtained via the plasmon dispersion relation of a two-dimensional electron gas (2DEG) which in the long wavelength limit reads: ${ }^{88}$

$$
\omega_{s p}^{(2 \mathrm{DEG})}(k)=\omega_{s p}^{(2 \mathrm{DEG})} \sqrt{Q}
$$

where $\omega_{s p}^{(2 \mathrm{DEG})}=\left(\omega_{v p} / \sqrt{2}\right) \sqrt{N_{2 \mathrm{D}} / N_{3 \mathrm{D}}}, N_{3 \mathrm{D}} \approx N_{2 \mathrm{D}} / t$ with $N_{2 \mathrm{D}}$ being an "effective" areal electronic density, and $t$ the inter-layer (or inter-wall in the case of MWCNTs) distance. We here assume $t=0.35 \mathrm{~nm}$ which is a typical interlayer distance in graphite. Note that, contrary to Eq. (11), the 2DEG (surface) plasmon has no cut-off frequency, i.e., $\omega_{s p}^{(2 \mathrm{DEG})}(Q=0)=0$. Instead, in the Q2D model the experimentally observed cut-off frequency value $\left(\sim \omega_{v p} / \sqrt{2}\right)$ is introduced (ad hoc) and, by expanding Eq. (13) at the small- $Q$ limit and combining with Eq. (10) under again the approximation $\varepsilon(\omega, k) \approx \varepsilon(\omega, Q)$, the following expression is obtained:

$$
\omega_{i}^{(\mathrm{Q} 2 \mathrm{D})}(k)=\sqrt{\omega_{i, 0}^{2}+\omega_{v p}^{2}(k t)^{1 / 2}+\beta^{2} k^{2}+k^{4} / 4}
$$

Thus, inclusion of boundary effects in the context of the Q2D model results in an additional dispersion term proportional to the square root of momentum transfer $(\sim \sqrt{k})$. In general, the approximation $\varepsilon(\omega, k) \approx \varepsilon(\omega, Q)$ is expected to be more correct near the surface than away from it, which is of course less of a problem for nanostructures than for standard bulk media. In order to obtain a general dispersion relation for use in our model dielectric function we will assume here that Eqs. (12) and (14) hold at all depths in the sample. This can be partly justified by the fact that for systems (e.g., long nanotubes and nanowires) where the electronic excitations are spatially confined in the transverse direction but (practically) not in the longitudinal one, the importance of the parallel component of $k$ is enhanced. ${ }^{51}$

In Fig. 3 we compare the plasmon line shift as predicted by the dispersion models denoted as 3D, BPS, and Q2D, and summarized, respectively, in Eqs. (10), (12), and (14), with the experimental EELS data ${ }^{81}$ for the $\pi+\sigma$ plasmon dispersion of bulk SWCNT samples, as well as with the recent IXS measurements ${ }^{49}$ on FWCNTs $(3 \pm 2$ walls $)$ and MWCNTs (14 \pm 5 walls). Although the comparison depicted in Fig. 3 is not straightforward because of the different type of nanotubes used in the experiments, we can see that the BPS model of Eq. (12) provides a fair representation of the data at $k \neq 0$ even when forced to reduce at $k=0$ to the (experimental) value used as input in our parameterized ELF model

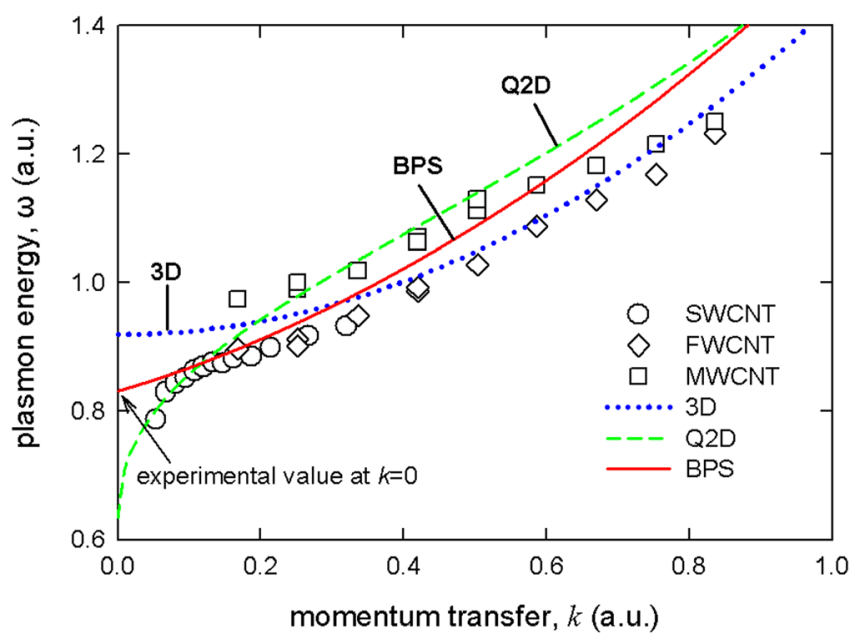

FIG. 3. (Color online) Comparison of momentum-dependent experimental data (symbols) with theoretical models for the $(\pi+\sigma)$ plasmon dispersion, $\omega(k)$. Circles: derived from EELS data ${ }^{81}$ for macroscopic samples of singlewalled CNT bundles; Diamonds: IXS data ${ }^{49}$ for few-walled CNTs ( $3 \pm$ walls); Boxes: IXS data ${ }^{49}$ for multi-walled CNTs ( $14 \pm 5$ walls). Dotted line: "3D" model calculations using Eq. (10); Full line: "BPS" model calculations using Eq. (12); Short dash: "Q2D" model calculations using Eq. (14). Note that the limiting value at $k \rightarrow 0$ denoted as "experimental value" is taken from the experimental data of Ref. 58 which are used to parameterize our model ELF at $k=0$. 
[Eq. (1)]. The analytic expressions of the 3D [Eq. (10)] and Q2D [Eq. (14)] models seem also capable of providing a reasonable fit to the data but, then, their plasmon value at $k=0$ is substantially larger or smaller, respectively, of the value used in our ELF model. Thus, for the present calculations, the BPS model provides the most consistent scheme for correcting the dispersion relation of infinite (i.e., unbounded) media.

\section{Influence of linewidth broadening}

In pure $2 \mathrm{D}$ and $3 \mathrm{D}$ systems and within the randomphase-approximation (RPA) plasmons are undamped excitations, i.e., they have infinite lifetime or zero linewidth, up to a critical wavevector $\left(k_{c}\right)$ where the plasmon energy enters into the electron-hole continuum and can decay in singleelectron excitations. ${ }^{90}$ The undamped approximation results in a sharp plasmon peak in the ELF (for $k<k_{c}$ ) which is in contrast to EELS and IXS experiments for a variety of different CNT systems that point out to a strong damping mechanism at all $k^{49,91}$ Although setting $\gamma \rightarrow 0$ might be inconsequential for the inelastic scattering of high-energy electrons, it can lead to sizable errors at low electron energies where only a small (or moderate) part of the ELF contributes to the Born inelastic scattering integral [see Eq. (17) below]. Thus, linewidth broadening effects in the ELF due to plasmon decay are expected to strongly influence inelastic scattering at low electron energies. In electron gas calculations the presence of damping is often treated in a phenomenological manner by introducing an empirical damping constant as exemplified by the Mermin dielectric function. ${ }^{92}$ A formal treatment of damping in the context of electron gas theory requires the evaluation of the dynamic (i.e., frequency-dependent) many-body local-field-correction (LFC) that accounts for (short-range) exchange and correlation effects. ${ }^{93}$ Unfortunately, no simple analytic expression of the dynamic LFC is available. On the other hand, damping is already included - in a phenomenological way - in our model through the Drude coefficients $\gamma_{i, 0}$ of Eq. (2) which essentially determine the width of the ELF at $k=0$. Although momentum broadening effects in the experimental EELS and IXS spectra of, respectively, vertically-aligned (VA) SWCNT bundles ${ }^{91}$ and MWCNTs (Ref. 49) are clearly visible with increasing $k$, it is hard to extract information on the dispersion of the plasmon linewidth from the experimental data due to overlapping features from different excitation peaks. However, a qualitative insight with respect to the $k$ dependence of the damping coefficient, $\gamma(k)$, can be gained by a simple phenomenological procedure whereby a singlepole representation [i.e., $m=1$ in Eqs. (1) and (2)] is fitted to the experimental data for the main energy-loss peak $(\pi+\sigma$ plasmon). In Fig. 4, the experimentally determined trends for the $k$-dependence of the plasmon linewidth for VA-SWCNT bundles and MWCNTs are compared against two analytic expressions, namely, the Mermin low- $k$ limit: ${ }^{85}$

$$
\gamma_{i}^{(\text {Mermin) }}(k)=\gamma_{i, 0}+\beta_{\mathrm{M}} k^{2}
$$

where $\beta_{\mathrm{M}}=(4 / 15) \gamma_{i, 0} v_{\mathrm{F}}^{2} / \omega_{v p}^{2}$, and the empirical formula: ${ }^{47}$

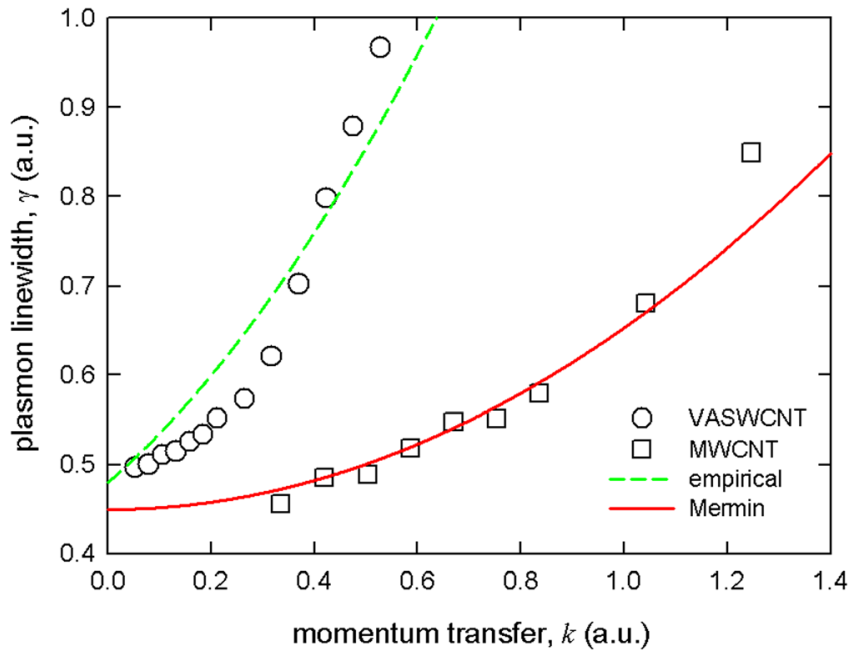

FIG. 4. (Color online) Comparison of experimental data (symbols) and theoretical calculations for the dispersion of the plasmon linewidth, $\gamma(k)$. Circles: EELS data for vertically-aligned (VA) SWCNT bundles, ${ }^{91}$ Boxes: IXS data for MWCNTs (Ref. 49). Full line: calculations using the Mermin expression of Eq. (15); Short dash: calculations using the empirical expression of Eq. (16).

$$
\gamma_{i}^{(\mathrm{emp})}(k)=\gamma_{i, 0}+\frac{k}{2}+\frac{k^{2}}{2}
$$

Although the data depicted in Fig. 4 should be considered qualitative, since the experimental spectra over the main plasmon peak region cannot be always reproduced accurately by a single-pole model, they can be helpful in guiding us toward a reasonable choice of the functional dependence of the damping coefficient $\gamma$ on momentum transfer $k$. We should first point out the significant difference between VASWCNTs and MWCNTs. Thus, the earlier established empirical formula [Eq. (16)] based on the VA-SWCNT data seem to be inappropriate for MWCNTs. Evidently, the Mermin expression described by Eq. (15) offers an improved representation of the MWCNT data over the depicted range of $k$.

\section{INELASTIC SCATTERING}

\section{A. Asymptotic bulk limit}

The plane-wave Born approximation (PWBA) provides a convenient theoretical framework for the present study since it is known to be reasonably valid for electrons above $\sim 50 \mathrm{eV}$ (Ref. 45). In fact, the PWBA underlines nearly all practical calculations of inelastic electron scattering in solids (e.g., the TPP formula ${ }^{45,83}$ ) leading to good agreement between experiment and calculations. In the non-relativistic PWBA the energy-loss differential cross section (DCS) for electrons moving through an unbounded (infinite) medium is given by the expression:

$$
\frac{d \Lambda}{d \omega}=\frac{1}{\pi E} \int_{k_{-}}^{k_{+}} \operatorname{Im}\left\{-\frac{1}{\varepsilon(\omega, k)}\right\} \frac{d k}{k}
$$

where $E$ is the electron kinetic energy and the limits of integration are $k_{ \pm}=\sqrt{2 E} \pm \sqrt{2(E-\omega)}$. As written, the magnitude of $\Lambda$ in Eq. (17) has dimensions of reciprocal length 
which makes it equivalent to the inverse inelastic mean free path; thus $d \Lambda / d \omega$ may also be called the differential inverse inelastic mean free path. In the present context, Eq. (17) represents the asymptotic bulk limit of the DCS which corresponds to electron trajectories sufficiently away from the surface inside the material where the electronic excitations can be adequately described by the bulk ELF, $\operatorname{Im}\{-1 / \varepsilon(\omega, k)\}$.

In Fig. 5 we present the asymptotic bulk limit of the DCS $(d \Lambda / d \omega)$ of MWCNTs for $1 \mathrm{keV}$ electrons over the 0 $100 \mathrm{eV}$ energy-loss range. This is the range where the inelastic scattering probability is largest due to energy losses to valence-electron excitations. The calculations are based on the bulk $\operatorname{ELF} \operatorname{Im}\{-1 / \varepsilon(\omega, k)\}$ of Eqs. (1) and (2) whereby the $k$ dependence is determined by different dispersion relations for the plasmon energy and linewidth. The calculations denoted by "3D", "BPS", and "Q2D" are based, respectively, on the plasmon dispersion relations Eqs. (10), (12), and (14) and the Mermin relation for damping, Eq. (15). The calculations denoted as "BPS-emp" and "BPS-const" use, respectively, the empirical damping expression, Eq. (16), or a constant damping, i.e., $\gamma_{i}(k)=\gamma_{i}(k=0)=\gamma_{i, 0}$.

It is clear from Fig. 5 that the three dispersion models for $\omega_{i}(k)$, represented by Eqs. (10), (12), and (14), predict significantly different DCSs. It is known from theory that the stronger the dispersion of the plasmon energy the more the DCS is shifted to higher energy losses with a smaller peak height. Thus, the use of a boundary correction to the standard dispersion relation of infinite (unbound) media which, according to the present analysis, results in a stronger dispersion mechanism has a sizable effect on the DCS and must be carefully considered. The dispersion of the plasmon linewidth has a much smaller effect on the DCS. It can be seen that the Mermin relation, Eq. (15), which predicts a smaller

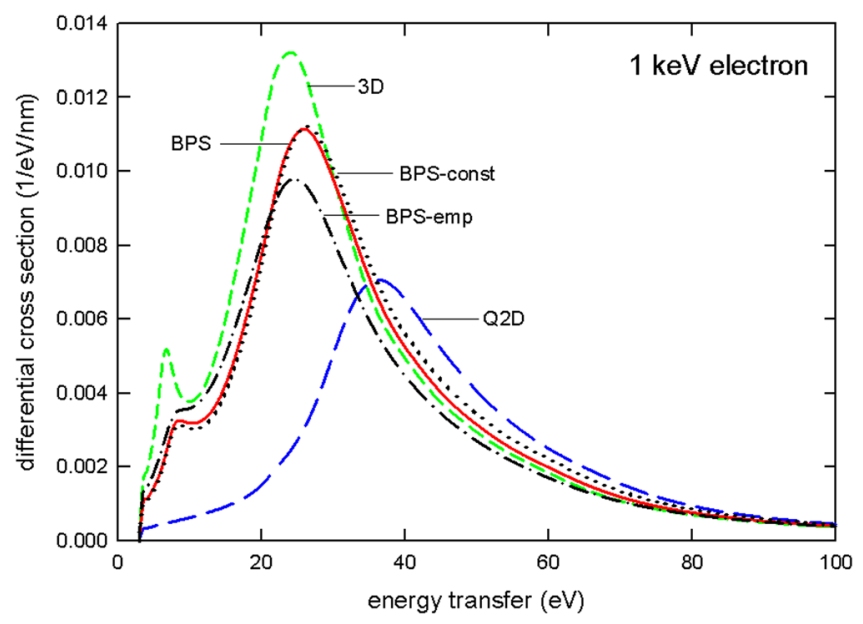

FIG. 5. (Color online) The asymptotic bulk limit of the energy-loss differential cross section (DCS) for inelastic scattering, Eq. (17), of $1 \mathrm{keV}$ electrons in MWCNTs using different dispersion relations for the plasmon energy and linewidth. The "3D" (short dash), "BPS" (full line), and "Q2D" (long dash) calculations employ Eqs. (10), (12), and (14), respectively, for the plasmon energy and the Mermin expression, Eq. (15), for the plasmon linewidth (damping); in the "BPS-emp" calculations (dash-dot line) the plasmon linewidth is described by the empirical expression Eq. (16) whereas in the "BPS-const" calculations (dotted line) a constant (non-dispersive) damping coefficient is used. linewidth broadening with $k$ than the empirical relation (see Fig. 4), results in the DCS being slightly shifted toward higher energy losses, as compared to using the empirical relation. This follows from the fact that a broader ELF favors low-energy losses (below the plasmon energy) and vice versa. However, at these relatively high electron energies (1 $\mathrm{keV}$ here), the use of a constant (non-dispersive) damping coefficient can reproduce the Mermin results quite well; the influence of damping increases though at lower electron energies as it will become apparent in the next figure.

In Fig. 6, we present the asymptotic bulk limit of the total cross section (TCS) for inelastic scattering as a function of electron energy $E$, which are obtained after integrating the DCS of Eq. (17) from $B_{i}=\omega_{\mathrm{th}, i}$ to $\left(E-B_{i}\right) / 2$. The setting of the upper integration limit to $\left(E-B_{i}\right) / 2$ instead of $E-B_{i}$ is due to the exchange effects between the incident and target electrons. Although we present results over a broad energy range $(10 \mathrm{eV} \leq E \leq 30 \mathrm{keV})$, those below $\sim 50 \mathrm{eV}$ are provided simply to illustrate the trend of the calculations and should be considered qualitative since the validity of Eq. (17) below $50 \mathrm{eV}$ is questionable. The neglect of relativistic effects in Eq. (17) also restrict the upper limit of electron energy to about $10-50 \mathrm{keV}$. The dispersion models used are the same that in Fig. 5. It is clear from Fig. 6 that the choice of the plasmon dispersion relation, $\omega_{i}(k)$, is also very important for the calculation of TCS. Specifically, the stronger the plasmon dispersion the smaller the TCS due to a larger fraction of ELF being outside the integration area in the $\omega-k$ plane [see Eq. (17)]. It is also evident in Fig. 6 that, by virtue of Bethe's theory, the effect of dispersion vanishes with increasing electron energy because of the dominant contribution of dipole $(k \approx 0)$ collisions in the inelastic scattering of high energy projectiles. For example, the inclusion of a boundary term (proportional to $k$ ) by means of the BPS model, see Eq. (12), reduces the "uncorrected" (i.e., 3D model) TCS by $25 \%, 10 \%$, and $5 \%$ at $0.1,1$, and $10 \mathrm{keV}$, respectively; the corresponding reduction resulting from the boundary term (proportional to $\sqrt{k}$ ) as described by the Q2D model, see Eq. (14), is $65 \%, 35 \%$, and $25 \%$. The plasmon

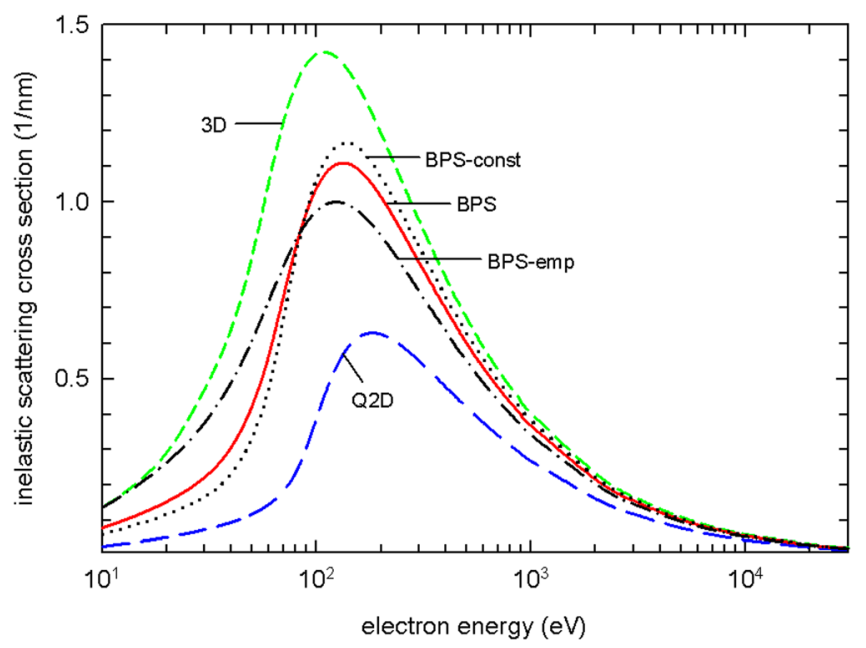

FIG. 6. (Color online) The asymptotic bulk limit of the total cross section (TCS) for inelastic scattering in MWCNTs as a function of electron energy, $E$, using different dispersion models (for explanation of models see Fig. 5). 
linewidth has also a noticeable effect on the TCS at not too high energies. In general, the broader the linewidth the smaller the TCS at energies above the peak maximum due to a larger fraction of ELF being outside the integration area in the $\omega-k$ plane [see Eq. (17)]. The opposite is true at low energies (below the peak maximum) where a broader ELF results in a larger TCS due to the enhancement of lower energy losses. Thus, compared to the constant (non-dispersed) damping calculations, the use of the Mermin expression, Eq. (15), increases the TCS at low-electron energies $(+27 \%$ at $50 \mathrm{eV})$ and reduces it at the region of the maximum $(-5 \%$ at $150 \mathrm{eV})$; the corresponding numbers for the empirical damping expression, Eq. (17), are much higher $(+83 \%$ at $50 \mathrm{eV}$ and $-15 \%$ at $150 \mathrm{eV})$.

According to the previous discussion, in what follows we will use the BPS [Eq. (12)] and Mermin [Eq. (15)] models for describing the dispersion of the plasmon energy and linewidth, respectively.

\section{B. Near surface region}

As the electron moves toward the MWCNT surface, the probability of surface excitations gradually dominates over that of bulk excitations. Due to the coupling between bulk and surface excitation modes that are orthogonal, the increased contribution of surface excitations to inelastic scattering in the near surface region results in a reduction of the corresponding bulk contribution, which is known as the Begrenzungs effect. ${ }^{67}$ On the other hand, as the electron approaches the surface from outside the material, practically only surface modes can be excited and, therefore, inelastic scattering would depend solely upon the surface ELF. In line with the assumption that curvature effects are small, we employ the Kwei-Wang-Tung (KWT) model ${ }^{94}$ for the spatially-varying DCS for the inelastic scattering of normally incident and escaping electrons from planebounded bulk media. According to the KWT model, energy losses to surface excitations are described by the surface ELF which is expressed in terms of the bulk dielectric function via Eq. (4). However, although Eq. (4) is strictly correct only for $k=0$, in the KWT model it is assumed to hold for all $k$ (the so-called step-density approximation). This is a standard approximation ${ }^{95}$ that should not cause any serious error in view of the fact that the $k$-dependence of the ELF is weak compared to the inverse dependence of the DCS upon $k$. One could also note that, for not too low electron energies, the integration over $k$ extends over a rather broad range, so the fine details in the dispersion of the surface ELF are not expected to be critical. Furthermore, it is natural to expect that the inclusion in the present work of boundary dispersion corrections due to surface excitations should further minimize the error made in the above approximation.

In the KWT model, an electron crosses (normally) the surface boundary between the "solid" $(z<0)$ and the "vacuum" $(z>0)$ at depth $z=0$ (where in our case the "solid" is the MWCNT). The direction of the electron trajectory can be either from solid to vacuum $(\mathrm{s} \rightarrow \mathrm{v})$ or from vacuum to solid ( $\rightarrow \mathrm{s}$ ). This distinction is important due to the different polarization forces acting on electrons moving towards to or away from the surface (see below). Then, the spatial variation of DCS for electrons escaping from $(s \rightarrow v)$ or incident to $(\mathrm{v} \rightarrow \mathrm{s})$ the surface can be calculated to a first approximation from the following expressions:

$$
\begin{aligned}
& \frac{d \Lambda^{(s-v)}}{d \omega} \approx \frac{2}{\pi} \int_{k_{-}}^{k_{+}} k d k \frac{v}{\omega^{2}+v^{2} k^{2}}\left\langle 2 \operatorname{Im}\left[\frac{-1}{\varepsilon(\omega, k)+1}\right] \exp (-k|z|)\right. \\
& \times\left[2 \cos \left(\frac{\omega z}{v}\right)-\exp (-k|z|)\right] \Theta(z)+\left\{2 \operatorname{Im}\left[\frac{-1}{\varepsilon(\omega, k)+1}\right]\right. \\
& \left.\left.\times \exp (-2 k|z|)+\operatorname{Im}\left[\frac{-1}{\varepsilon(\omega, k)}\right][1-\exp (-2 k|z|)]\right\} \Theta(-z)\right\rangle
\end{aligned}
$$

$$
\begin{aligned}
& \frac{d \Lambda^{(v-s)}}{d \omega} \approx \frac{2}{\pi} \int_{k_{-}}^{k_{+}} k d k \frac{v}{\omega^{2}+v^{2} k^{2}}\left\langle 2 \operatorname{Im}\left[\frac{-1}{\varepsilon(\omega, k)+1}\right]\right. \\
& \times \exp (-2 k|z|) \Theta(z)+\left\{2 \operatorname{Im}\left[\frac{-1}{\varepsilon(\omega, k)+1}\right] \exp (-k|z|)\right. \\
& {\left[2 \cos \left(\frac{\omega z}{v}\right)-\exp (-k|z|)\right]+[1+\exp (-2 k|z|)-2 \exp (-k|z|)} \\
& \left.\left.\left.\times \cos \left(\frac{\omega z}{v}\right)\right] \operatorname{Im}\left[\frac{-1}{\varepsilon(\omega, k)}\right]\right\} \Theta(-z)\right\rangle
\end{aligned}
$$

where $v$ is the electron velocity $\left(E=v^{2} / 2\right)$. The integration limits in Eqs. (18a) and (18b) are $k_{ \pm}=\sqrt{q_{ \pm}^{2}-(\omega / v)^{2}}$ with $q_{ \pm}=\sqrt{2 E} \pm \sqrt{2(E-\omega)}$. We should note that in the derivation of Eqs. (18a) and (18b) it is assumed that $\varepsilon(\omega, k) \approx \varepsilon(\omega, Q)$. This approximation, already used in Eqs. (12) and (14), has been shown to work well for the near surface region and, accordingly, Eqs. (18a) and (18b) should be more correct near the surface than away from it. ${ }^{95}$ This deficiency of the model manifests itself at relatively large distances from the surface inside the material where, for example, Eqs. (18a) and (18b) do not reduce to the asymptotic bulk expression, Eq. (17). Obviously, this deficiency is less of a problem for nanostructures. An improved model with the correct asymptotic properties valid over all angular orientations of the electron trajectory is available ${ }^{96}$ but with the cost of a substantial increase in computation. ${ }^{97}$

Although a separation of bulk and surface losses is not strictly correct, the KWT model offers a first approximation to the effect of surface losses upon the DCS. Specifically, the terms including $\operatorname{Im}\{-1 / \varepsilon(\omega, k)\}$ represent energy losses to bulk excitations whereas those including $\operatorname{Im}\{-1 /[\varepsilon(\omega, k)$ $+1]\}$ represent energy losses to surface excitations. For electrons traveling in the near surface region from inside the solid, Eq. (18a) predicts a surface-correction to the bulk excitation probability (Begrenzungs effect) that decreases exponentially with distance from the surface in line with recent findings. ${ }^{98}$ The pure surface contribution also decreases exponentially with depth in the material while being maximum at the surface. Note that when electrons are outside the material $(z>0)$, Eqs. (18a) and (18b) contain no contribution from bulk losses but a non-vanishing contribution from surface losses. 


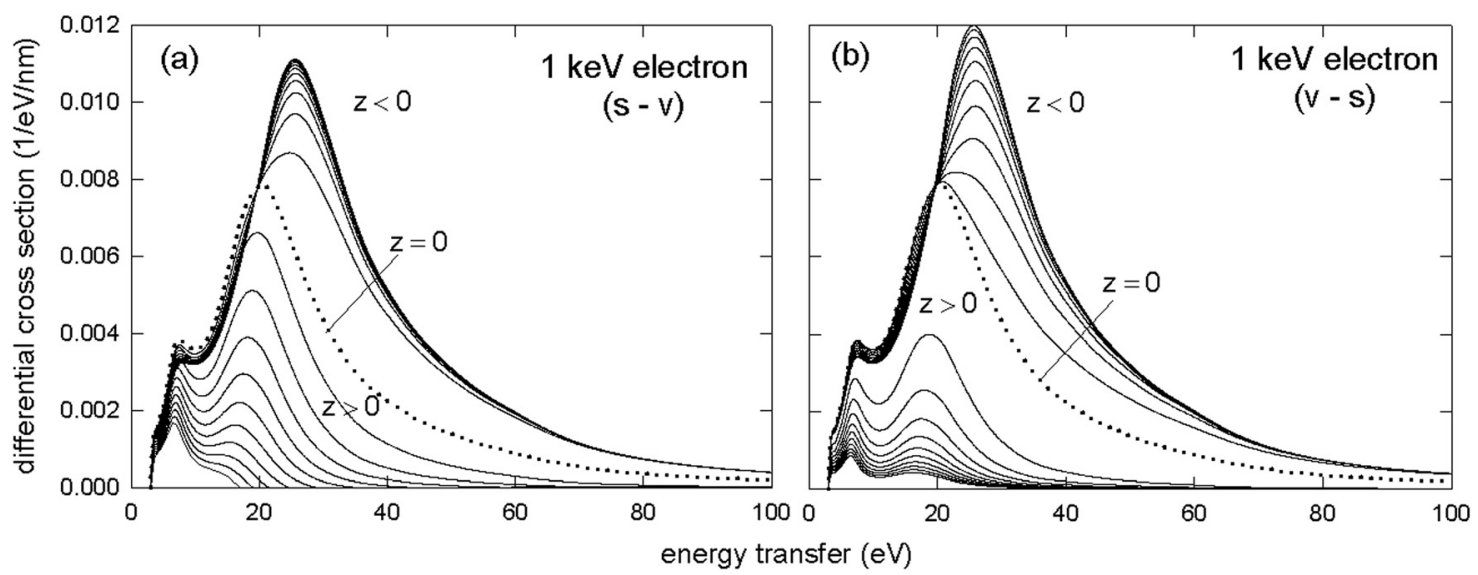

FIG. 7. Position-dependent differential cross section (DCS) for the inelastic scattering of $1 \mathrm{keV}$ electrons through MWCNTs: top curve corresponds to $1 \mathrm{~nm}$ depth inside the material $(\mathrm{z}=-10 \AA$ ) and bottom curve to $1 \mathrm{~nm}$ outside the material ( $\mathrm{z}=+10 \AA$ ). The curves from top to bottom are in steps of $1 \AA$. Panel (a) pertains to escaping (s-v) electron trajectories whereas panel (b) to incident (v-s) electron trajectories.

In Fig. 7, we present the DCS for $1 \mathrm{keV}$ electrons at different positions relative to the MWCNT surface for both escaping (s-v) and incident ( $\mathrm{v}-\mathrm{s})$ trajectories. It can be seen that the DCS is very sensitive to the position of the electron in the near surface region. In particular, the DCS outside the material or in the shallow region inside of it, exhibits important differences - both in shape and intensity - with the (asymptotic) bulk DCS which is well approximated by the top curve in Fig. 7 (for further depths inside the material the DCS remains practically unchanged). These changes in the DCS are the result of the interplay of bulk and surface losses and may have important consequences in theoretical models of electron transport in MWCNT systems. ${ }^{28,43}$ For electron trajectories in the near surface region the DCS shows an enhancement of low energy losses due to the increased contribution of surface excitations relative to bulk excitations. The effect is of course most evident for trajectories outside the material where only surface excitations are possible. We can also see that the DCS depends on the electron direction (incident or escaping) for equal distances from the surface. This effect is due to the different polarization forces acting on the electron from the bulk and surface induced charges. A simple illustration of the above effect is presented in Fig. 8 where we depict the bulk and surface contribution to the DCS for escaping (s-v) and incident (v-s) electrons at a depth of $1 \AA$ inside the material (i.e., $\mathrm{z}=-1 \AA$ ). We can see that, at equal depths inside the material, the escaping electron (s-v) has a smaller surface contribution (than the incident electron, $\mathrm{v}$-s) due to an accelerating force caused by the polarization of surface charges (this force is de-accelerating in the $\mathrm{v}$-s case) but this reduction is more than compensated by a larger bulk contribution due to the retarding force from the polarization of bulk charges. However, in both cases, and in accordance to Eq. (18a) and (18b), with increasing depth inside the material the contribution of surface losses to the DCS rapidly diminishes whereas that from bulk losses increases until, eventually, the total DCS approaches its asymptotic bulk limit described by Eq. (17). This is illustrated in Fig. 9 for the case of incident electron trajectories (v-s). Clearly, the surface contribution, which is the sole contribution to the DCS at $z=0$, diminishes rapidly with depth whereas the opposite is true for the bulk contribution. The latter is nearly equivalent to the asymptotic bulk DCS at $1 \mathrm{~nm}$ depth inside the material (i.e., at $\mathrm{z}=-10 \AA$ ).

In Fig. 10, we show the depth-dependence of the TCS for the inelastic scattering of $1 \mathrm{keV}$ electron in both the incident (v-s) and escaping (s-v) geometry obtained by integration of Eqs. (18a) and (18b) over energy transfer. The bulk and surface contributions to the total TCS is separately depicted. We can see that for both geometries the bulk contribution reaches its asymptotic value at $\sim 1-2 \mathrm{~nm}$ depth. The surface contribution is seen to rise rapidly in the region very

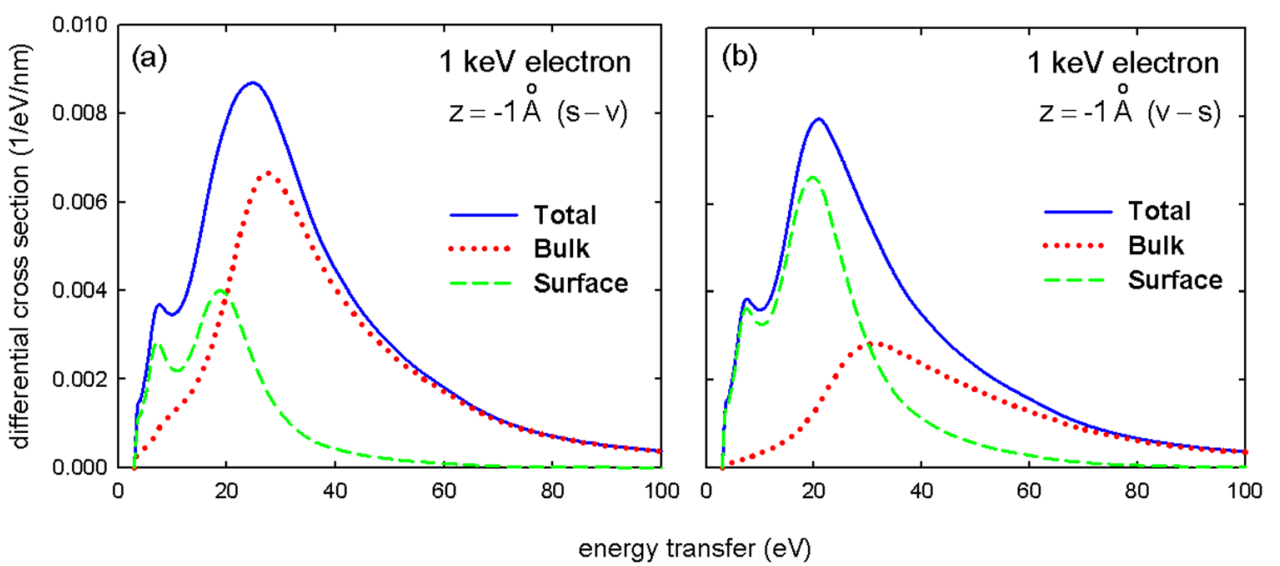

FIG. 8. (Color online) Position-dependent differential cross section (DCS) for the inelastic scattering of $1 \mathrm{keV}$ electron at a depth of $1 \AA$ inside $(\mathrm{z}=-1 \AA$ ) the MWCNT with the bulk and surface contribution explicitly shown. Panel (a) pertains to escaping (s-v) electron trajectories whereas panel (b) to incident $(\mathrm{v}-\mathrm{s})$ electron trajectories. 


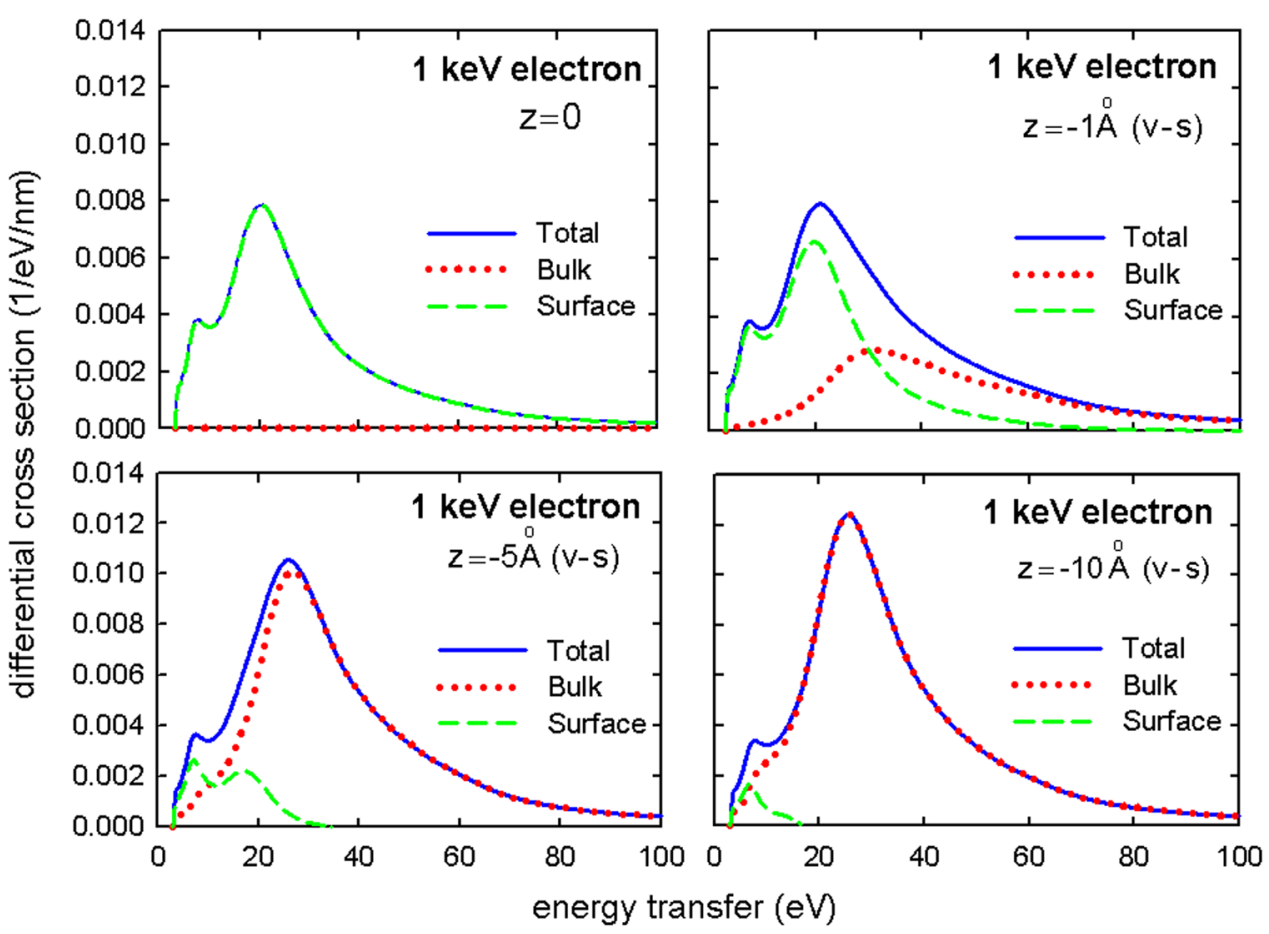

FIG. 9. (Color online) Position-dependent differential cross section (DCS) for the inelastic scattering of $1 \mathrm{keV}$ incident electron (v-s) at different depths inside the MWCNT with the bulk and surface contributions to the DCS explicitly shown. close to the surface inside the material while decreasing exponentially outside. On the other hand, the bulk contribution exhibits a steep drop near the surface (Begrenzungs effect) due to the coupling of bulk and surface modes which are orthogonal, and vanishes outside the material. Evidently, the general notion of a spatially invariant electron inelastic mean free path inside a solid seems to apply also to the present case despite the fact that, as shown in Figs. 7-9, the excitation mechanism for electrons in the near surface region changes drastically.

\section{SUMMARY}

We have presented a simple methodology for including bulk and surface excitation effects in the inelastic scattering of low-energy electrons $(10 \mathrm{eV}-30 \mathrm{keV})$ in MWCNTs. The contribution of bulk and surface losses along with the Begrenzungs effect are treated in a unified manner using a semiempirical dielectric response function model with parameters adjusted to experimental spectroscopy data for MWCNTs. Boundary corrections to the standard dispersion relations used to extend the energy loss function (ELF) of infinite media to arbitrary values of momentum transfer are suggested using simple arguments from electron gas theory. The influence of various dispersion approximations to the energy-loss differential and total inelastic scattering cross sections is quantified. The spatial variation of inelastic scattering with distance from the surface is examined for both incident and escaping electron trajectories normal to the surface. The general notion of a spatially invariant inelastic mean free path inside the material is shown to be a good approximation for MWCNTs. However, the excitation mechanism is shown to be changing drastically in the near surface region significantly modifying the shape and intensity of the energy-loss differential inelastic scattering cross section. The present work is expected to be useful in various applications

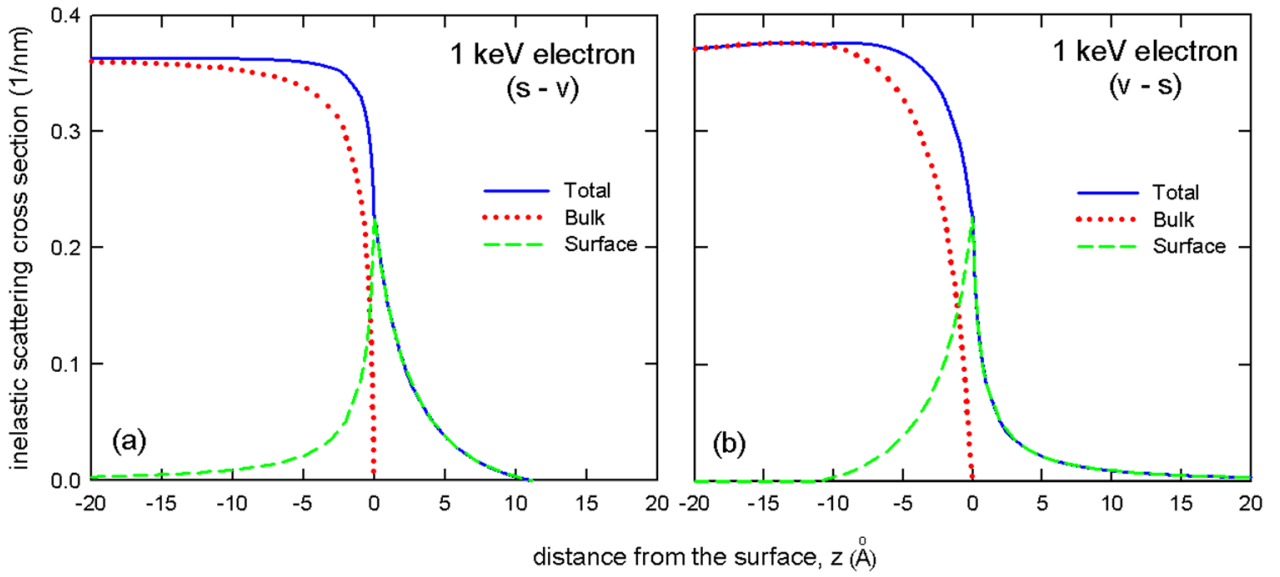

FIG. 10. (Color online) Position-dependent total cross section (TCS) for the inelastic scattering of $1 \mathrm{keV}$ electrons by MWCNTs with the bulk and surface contributions explicitly shown. Panel (a) pertains to escaping (s-v) electron trajectories whereas panel (b) to incident $(\mathrm{v}$-s) electron trajectories. 
whereby low-energy electron beam irradiation effects in MWCNTs play an important role such as, for example, in the use of SEM and EBL for beam-assisted engineering of nanodevices as well as in the characterization, fabrication, and patterning of CNT-FETs. It is envisioned that by providing a computationally tractable, yet realistic scheme, of inelastic scattering induced electronic excitations in MWCNTs, the present work will also be useful to the development of Monte Carlo electron transport simulation codes specific to this system. Finally, we should point out that due to the restrictions of the Born approximation it is not clear if the present methodology will be adequate for too low electron energies (say below $\sim 50 \mathrm{eV}$ ) and, in general, it should be more correct for large rather than small diameter MWCNTs (say with more than 15 walls or diameter larger than $10 \mathrm{~nm}$ ) due to the neglect of curvature effects.

\section{ACKNOWLEDGMENTS}

We are grateful to Mary Upton and Christian Kramberger for kindly providing to us raw data and useful clarifications regarding their IXS and EELS measurements of Refs. 49 and 91, respectively. Financial support of D.E., I.K., and K.K. by the European Union FP7 ANTICARB (Grant No. HEALTH-F2-2008-201587) and of I.A. and RGM by the Spanish Ministerio de Ciencia e Innovación (Project FIS2010-17225) is acknowledged.

${ }^{1}$ S. Iijima, Nature 354, 56 (1991).

${ }^{2}$ Carbon Nanotubes, Synthesis, Structure, Properties and Applications, edited by M. S. Dresselhaus, G. Dresselhaus, and P. Avouris (Springer, Berlin, 2001).

${ }^{3}$ A. V. Krasheninnikov and F. Banhart, Nat. Mater. 6, 723 (2007).

${ }^{4}$ A. V. Krasheninnikov and K. Nordlund, J. Appl. Phys. 107, 071301 (2010).

${ }^{5}$ W.-K. Hong, C. Lee, D. Nepal, K. E. Geckeler, K. Shin, and T. Lee, Nanotechnology 17, 5675 (2006).

${ }^{6}$ P. J. Boul, K. Turner, J. Li, M. X. Pulikkathara, R. C. Dwivedi, E. D. Sosa, Y. Lu, O. V. Kuznetsov, P. Moloney, R. Wilkins, M. J. O'Rourke, V. N. Khabashesku, S. Arepalli, and L. Yowell, J. Phys. Chem. C. 113, 14467 (2009).

${ }^{7}$ X. W. Tang, Y. Yang, W. Kim, Q. Wang, P. F. Qi, H. J. Dai, and L. Xing, Phys. Med. Biol. 50, N23 (2005).

${ }^{8}$ S. Y. Hong, G. Tobias, K. T. Al-Jamar, B. Ballesteros, H. Ali-Boucetta, S. Lozano-Perez, P. D. Nellist, R. B. Sim, C. Finucane, S. J. Mather, M. L. H. Green, K. Kostarelos, and B. G. Davis, Nat. Mater. 9, 485 (2010).

${ }^{9}$ F. Banhart, Rep. Prog. Phys. 62, 1181 (1999).

${ }^{10}$ B. W. Smith and D. E. Luzzi, J. Appl. Phys. 90, 3509 (2001).

${ }^{11}$ A. V. Krasheninnikov, F. Banhart, J. X. Li, A. S. Foster, and R. M. Nieminen, Phys. Rev. B 72, 125428 (2005)

${ }^{12}$ J. H. Warner, F. Schaffel, G. Zhong, M. H. Rummeli, B. Buchner, J. Robertson, and G. A. D. Briggs, ACS Nano. 3, 1557 (2009).

${ }^{13}$ T. D. Yuzvinsky, A. M. Fennimore, W. Mickelson, C. Esquivias, and A. Zettl, Appl. Phys. Lett. 86, 053109 (2005).

${ }^{14}$ J. Martinez, T. D. Yuzvinsky, A. M. Fennimore, A. Zettl, R. Garcia, and C. Bustamante, Nanotechnology 16, 2493 (2005).

${ }^{15}$ S. Suzuki, D. Takagi, Y. Homma, and Y. Kobayashi, Jpn. J. Appl. Phys. 44, L133 (2005).

${ }^{16}$ P. Liu, F. Arai, and T. Fukuda, Appl. Phys. Lett. 89, 113104 (2006).

${ }^{17}$ K. Molhave, S. B. Gudnason, A. T. Pedersen, C. H. Clausen, A. Horsewell, and P. Boggild, Ultramicroscopy 108, 52 (2007).

${ }^{18}$ S. Suzuki, K. Yamaya, Y. Homma, and Y. Kobayashi, Carbon 48, 3211 (2010).

${ }^{19}$ S. Suzuki and Y. Kobayashi, Chem. Phys. Lett. 430, 370 (2006).

${ }^{20}$ A. Nojeh, B. Shan, and R. F. W. Pease, Phys. Rev. Lett. 96, 056802 (2006).

${ }^{21}$ S. Suzuki and Y. Kobayashi, Jpn. J. Appl. Phys. 47, 2040 (2008).

${ }^{22}$ A. V. Krasheninnikov, J. Comput. Theor. Nanosci. 5, 1 (2008).
${ }^{23}$ K. Kanzaki, S. Suzuki, H. Inokawa, Y. Ono, A. Vijayaraghavan, and Y. Kobayashi, J. Appl. Phys. 101, 034317 (2007).

${ }^{24}$ G. Rius, J. Llobet, M. J. Esplandiu, L. Sole, X. Borrise, and F. PerezMurano, Microelectron. Eng. 86, 892 (2009)

${ }^{25}$ J. Chan, B. Burke, M. Cabral, C. Hu, J. Campbell, L. Harriott, and K. A Williams, J. Phys.: Condens. Matter. 22, 334212 (2010).

${ }^{26}$ A. Nojeh, W.-K. Wong, E. Yieh, and R. F. Pease, J. Vac. Sci. Technol. B 22, 3124 (2004).

${ }^{27}$ M. Michan, P. Yaghoobi, B. Wong, and A. Nojeh, Phys. Rev. B 81, 195438 (2010).

${ }^{28}$ M. K. Alam, P. Yaghoobi, and A. Nojeh, Scanning 31, 1 (2010).

${ }^{29}$ J. Luo, J. H. Warner, C. Feng, Y. Yao, Z. Jin, H. Wang, C. Pan, S. Wang, L. Yang, Y. Li, J. Zhang, A. A. R. Watt, L.-M. Peng, J. Zhu, and G. A. D. Briggs, Appl. Phys. Lett. 96, 213113 (2011).

${ }^{30}$ C. J. Powell, Surf. Sci. 299, 34 (1994).

${ }^{31}$ S. Tougaard and F. Yubero, Surf. Interface Anal. 36, 824 (2004).

${ }^{32}$ V. A. Basiuk, K. Kobayashi, T. Kaneko, Y. Negishi, E. V. Basiuk, and J.-M. Saniger-Blesa, Nano Lett. 2, 789 (2002).

${ }^{33}$ B. Khare, M. Meyyappan, M. H. Moore, P. Wilhite, H. Imanaka, and B. Chen, Nano Lett. 3, 643 (2003).

${ }^{34}$ C. D. Cress, C. M. Schauerman, B. J. Landi, S. R. Messenger, R. P. Raffaelle, and R. J. Walters, J. Appl. Phys. 107, 014316 (2010).

${ }^{35}$ B. Ziaja, R. A. London, and J. Hajdu, J. Appl. Phys. 99, 033514 (2006).

${ }^{36}$ Y. Mera, Y. Harada, S. Arima, K. Hata, S. Shin, and K. Maeda, Chem. Phys. Lett. 473, 138 (2009).

${ }^{37}$ T. Stockli, J. M. Bonard, A Chatelain, Z. L. Wang, and P. Stadelmann, Appl. Phys. Lett. 80, 2982 (2002).

${ }^{38}$ Y.-N. Wang and Z. L. Miskovic, Phys. Rev. A. 66, 042904 (2002).

${ }^{39}$ A. Balassis and G. Gumbs, Phys. Rev. B 74, 045420 (2006).

${ }^{40}$ S. W. Chung, D. J. Mowbray, Z. L. Miskovic, F. O. Goodman, and Y.-N. Wang, Radiat. Phys. Chem. 76, 524 (2007).

${ }^{41}$ S. Segui, J. L. Gervasoni, and N. R. Arista, Radiat. Phys. Chem. 76, 582 (2007).

${ }^{42}$ D. J. Mowbray, S. Segui, J. Gervasoni, Z. L. Miskovic, and N. R. Arista, Phys. Rev. B 82, 035405 (2010).

${ }^{43}$ M. K. Alam, P. Yaghoobi, and A. Nojeh, J. Vac. Sci. Technol. B 28, C6J13 (2010).

${ }^{44}$ C. J. Powell, Surf. Sci. 44, 29 (1974).

${ }^{45}$ C. J. Powell and A. Jablonski, J. Phys. Chem. Ref. Data. 28, 19 (1999).

${ }^{46}$ Y. H. Tu, C. M. Kwei, and C. J. Tung, Surf. Sci. 600, 820 (2006).

${ }^{47}$ I. Kyriakou, D. Emfietzoglou, R. Garcia-Molina, I. Abril, and K. Kostarelos, Appl. Phys. Lett. 94, 263113 (2009).

${ }^{48}$ D. Emfietzoglou, I. Kyriakou, R. Garcia-Molina, I. Abril, and K. Kostarelos, J. Appl. Phys. 108, 054312 (2010).

${ }^{49}$ M. H. Upton, R. F. Klie, J. P. Hill, T. Gog, D. Casa, W. Ku, Y. Zhu, M. Y. Sfeir, J. Misewich, G. Eres, and D. Lowndes, Carbon 47, 162 (2009).

${ }^{50}$ R. F. Egerton, Electron Energy-Loss Spectroscopy in the Electron Microscope Plenum,New York, 1989.

${ }^{51}$ N. Zabala, E. Ogando, A. Rivacoba, and F. J. Garcia de Abajo, Phys. Rev. B. 64, 205410 (2001).

${ }^{52}$ M. Kociak, L. Henrard, O. Stéphan, K. Suenaga, and C. Colliex, Phys. Rev. B 61, 013936 (2000).

${ }^{53}$ A. G. Marinopoulos, L. Reining, A. Rubio, and N. Vast, Phys. Rev. Lett. 91, 046402 (2003).

${ }^{54}$ A. G. Marinopoulos, L. Wirtz, A. Marini, V. Olevano, A. Rubio, and L. Reining, Appl. Phys. A 78, 1157 (2004).

${ }^{55}$ A. G. Marinopoulos, L. Reining, and A. Rubio, Phys. Rev. B 78, 235428 (2008).

${ }^{56}$ A. G. Marinopoulos, L. Reining, V. Olevano, and A. Rubio, Phys. Rev. Lett. 89, 076402 (2002).

${ }^{57}$ E. Lidorikis and A. C. Ferrari, ACS Nano. 3, 1238 (2009).

${ }^{58}$ R. Kuzuo, M. Terauchi, and M. Tanaka, Jpn. J. Appl. Phys. 31, L1484 (1992).

${ }^{59}$ C. T. Chantler, K. Olsen, R. A. Dragoset, A. R. Kishore, S. A. Kotochigova, and D. S. Zucker, http://physics.nist.gov/ffast.

${ }^{60}$ R.H. Ritchie and A. Howie, Philos. Mag. 36, 463 (1977).

${ }^{61}$ The empirical Drude coefficients of our model are provided by the authors upon request.

${ }^{62}$ S. Tanuma, C. J. Powell, and D. R. Penn, J. Electron Spectrosc. Relat. Phenom. 62, 95 (1993).

${ }^{63}$ H. Venghaus, Phys. Stat. Solidi. B 71, 609 (1975).

${ }^{64}$ U. Buchner, Phys. Stat. Solidi. B 81, 227 (1976).

${ }^{65}$ P. M. Ajayan, S. Iijima, and T. Ichihashi, Phys. Rev. B 47, 6859 (1993). 
${ }^{66}$ L. A. Bursill, P. A. Stadelmann, J. L. Peng, and S. Prawer, Phys. Rev. B 49, 2882 (1994).

${ }^{67}$ R. H. Ritchie, Phys. Rev. 106, 874 (1957).

${ }^{68}$ C. D. Powell and J. B. Swan, Phys. Rev. 115, 869 (1959).

${ }^{69}$ T. Stockli, J.-M. Bonard, P-.A. Stadelmann, and A. Chatelain, Z. Phys. D 40, 425 (1997).

${ }^{70}$ B. W. Reed and M. Sarikaya, Phys. Rev. B 64, 195404 (2001).

${ }^{71}$ O. Stéphan, M. Kociak, L. Henrard, K. Suenaga, A. Gloter, M. Tence, E. Sandre, and C. Colliex, J. Electron Spectrosc. Related Phenom. 114, 209 (2001).

${ }^{72}$ O. Stéphan, D. Taverna, M. Kociak, K. Suenaga, L. Henrard, and C. Colliex, Phys. Rev. B 66, 155422 (2002).

${ }^{73}$ Z. L. Wang, Micron 27, 265 (1996).

${ }^{74}$ S. Segui, J. L. Gervasoni, and N. R. Arista, Surf. Sci. 601, 4169 (2007).

${ }^{75}$ C. Yannouleas, E. N. Bogachek, and U. Landman, Phys. Rev. B 53, 10225 (1996).

${ }^{76}$ J. L. Gervasoni, S. Segui, and N. Arista, Radiat. Eff. Defects Solids. 162, 267 (2007)

${ }^{77}$ J. L. Gervasoni, S. Segui, and N. Arista, Vacuum. 84, 262 (2007).

${ }^{78}$ J. L. Gervasoni, Nucl. Instr. Meth. B 267, 235 (2009).

${ }^{79}$ M. Z. Herrera and J. L. Gervasoni, Nucl. Instrum. Methods Phys. Res. B 267, 415 (2009).

${ }^{80}$ I. Villó-Pérez, N. R. Arista, Surf. Sci. 603, 1 (2009).

${ }^{81}$ T. Pichler, M. Knupfer, M. S. Golden, J. Fink, A. Rinzler, and R. E. Smalley, Phys. Rev. Lett. 80, 4729 (1998).
${ }^{82}$ J. M. Fernandez-Varea, R. Mayol, D. Liljequist, and F. Salvat, J. Phys.: Condens. Matter 5, 3593 (1993).

${ }^{83}$ S. Tanuma, C. J. Powell, and D. R. Penn, Surf. Interface Anal. 43, 689 (2011).

${ }^{84}$ J. C. Ashley, J. Electron Spectrosc. Relat. Phenom. 28, 177 (1982).

${ }^{85}$ K. Sturm, Adv. Phys. 31, 1 (1982).

${ }^{86}$ P. M. Echenique, R. H. Ritchie, and W. Brandt, Phys. Rev. B 20, 2567 (1979).

${ }^{87}$ R. H. Ritchie, Prog. Theor. Phys. 29, 607 (1963).

${ }^{88}$ L. Kong, B. Yan, and X. Hu, Plasma Sci. Technol. 9, 519 (2007).

${ }^{90}$ M. Dressel and G. Grüner, Electrodynamics of Solids (Cambridge University Press, Cambridge, 2002).

${ }^{91}$ C. Kramberger, R. Hambach, C. Giorgetti, M. H. Rummeli, M. Knupfer, J. Fink, B. Buchner, L. Reining, E. Einarsson, S. Maruyama, F. Sottile, K. Hannewald, V. Olevano, A. G. Marinopoulos, and T. Pichler, Phys. Rev. Lett. 100, 196803 (2008).

${ }^{92}$ N. D. Mermin, Phys. Rev. B 1, 2362 (1970).

${ }^{93}$ K. Sturm and A. Gusarov, Phys. Rev. B 62, 16474 (2000).

${ }^{94}$ C. M. Kwei, C. Y. Wang, C. J. Tung, Surf. Interface Anal. 26, 682 (1998).

${ }^{95}$ F. Yubero, J. M. Sanz, B. Ramskov, and S. Tougaard, Phys. Rev. B 53, 9719 (1996)

${ }^{96}$ Y. C. Li, Y. H. Tu, C. M. Kwei, and C. J. Tung, Surf. Sci. 589, 67 (2005).

${ }^{97}$ M. Novák, Surf. Sci. 602, 1458 (2008).

${ }^{98}$ N. Pauly, S. Tougaard, Surf. Sci. 603, 2158 (2009). 